\title{
A functional polymorphism in the pre-miR-146a gene influences the prognosis of glioblastoma multiforme by interfering with the balance between Notch1 and Notch2
}

\author{
RONGYAO LIU*, WEIHUA LI* and CHUNMING WU \\ Department of Neurosurgery, The First Affiliated Hospital of Dalian Medical University, Dalian, Liaoning 116011, P.R. China
}

Received April 21, 2014; Accepted January 22, 2015

DOI: $10.3892 / \mathrm{mmr} .2015 .4067$

\begin{abstract}
The aim of the present study was to evaluate the association between a polymorphism (rs2910164) in the microRNA (miR)-146a precursor and the prognosis of glioblastoma multiforme (GBM), as well as to examine the possible underlying mechanism in a Chinese population. A total of 380 patients with histologically confirmed GBM were recruited between 2008 and 2012, and were genotyped for the rs2910164 polymorphism using Sanger sequencing. The Kaplan-Meier method was used to estimate overall survival (OS), and univariate and multivariate Cox proportional hazard regression analyses were used to evaluate the effect of miR-146a polymorphisms on OS. It was identified that the rs2910164 CC genotype was significantly associated with a decreased OS among the patients with GBM $(\mathrm{P}=0.002)$. It was confirmed that Notch1 and Notch2 were targets of miR-146a and it was demonstrated that the introduction of miR-146a mimic suppressed the levels of Notch1 and Notch2 to different extents, resulting in a reduced Notch1/Notch2 ratio with an increase in miR-146a mimic concentration in U251 cells. Additionally, resected tumor specimens were collected from $138 \mathrm{GBM}$ patients and the expression levels of miR-146a, Notch1 and Notch2 were examined using reverse transcription-quantitative polymerase chain reaction and western blot analysis. Consistent with the in vitro study, lower levels of miR-146a, higher levels of Notch1 and Notch2, and a higher Notch1/Notch2 ratio were identified in the CC genotype group compared with those of the GG/GC group. In the present study, the rs2910164 C allele was found to be associated with a reduced survival rate in patients with GBM, and the observed association between the $\mathrm{CC}$ genotype and poorer prognosis of
\end{abstract}

Correspondence to: Dr Chunming Wu, Department of Neurosurgery, The First Affiliated Hospital of Dalian Medical University, 222 Zhongshan Road, Dalian, Liaoning 116011, P.R. China

E-mail: chunmingwu001@163.com

*Contributed equally

Key words: microRNA-146a, Notch, polymorphism, prognosis, glioma
GBM was at least partially mediated by the decreased expression of miR-146a, which interfered with the balance of Notch1 and Notch2.

\section{Introduction}

A glioma is a type of tumor that originates from glial cells (astrocytes or oligodendrocytes), which are non-neuronal supporting cells. Gliomas are the most fatal type of brain tumor, and glioblastoma multiforme (GBM) is the most common and aggressive subtype of glioma in humans (1). Although the etiology of the tumor remains to be elucidated, GBM is hypothesized to occur as a result of the interaction between multiple genetic and environmental factors. Genetic factors have been reported to contribute to gliomagenesis, as evidenced by familial aggregation studies $(2,3)$, as well as rare Mendelian syndromes, including neurofibromatosis and Turcot syndrome (4). Associations have been established between GBM susceptibility and single nucleotide polymorphisms (SNPs) in genes that were independently identified to be candidate genes involved in gliomagenesis in two genome-wide association studies (5-7).

MicroRNAs (miRNAs/miRs) are a class of 22 -nucleotide-long non-protein coding RNAs, which are hypothesized to regulate up to a third of all protein-coding genes via binding to the 3' untranslated region (UTR) of target gene messenger RNA (mRNA), resulting in translational repression and/or degradation of mRNA (8). miRNAs have previously been reported to be involved in the regulation of various biological processes, including the cell cycle, cell differentiation, apoptosis and metastasis (8). Accumulating lines of evidence have demonstrated that SNPs or mutations in the miRNA sequence may influence cancer susceptibility via the alteration of miRNA expression, maturation or miRNA-miRNA interactions $(9,10)$. An association between aberrant miRNA expression and oncogenesis has been consistently reported $(11,12)$, and the dysregulated expression of miRNAs and their targets is hypothesized to occur as a result of functional polymorphisms in the miRNA sequence (13-17). The rs2910164 polymorphism in the miR-146a precursor has previously been associated with various types of cancer, including breast and ovarian cancer $(18,19)$, papillary thyroid cancer (11), prostate cancer (20), esophageal squamous cell carcinoma (21) and gastric (22) and hepatocellular cancer (23). 
Jazdzewski et al (11) presented evidence demonstrating that the $\mathrm{C}$ allele of rs2910164 may induce a reduction in mature miR-146a levels and decreased inhibition of its target genes, including tumor necrosis receptor-associated factor 6 (TRAF6) and interleukin-1 receptor-associated kinase 1 (IRAK1) by interfering with the processing of pre-microRNA.

Notch signaling comprises a major pathway involved in GBM development. Notch signaling has been observed to maintain the proliferation of normal neural precursor cells and has been defined as a survival marker in gliomas, as indicated by its overexpression in glioma tissue and ability to promote glioma cell migration and invasion by stimulation of $\beta$-catenin and nuclear factor $-\kappa \mathrm{B}(\mathrm{NF}-\kappa \mathrm{B})$ signaling via AKT activation $(15,16)$. Xu et al $(21)$ revealed that two major members of the Notch family, Notch1 and Notch2, have opposite roles in regulating the proliferation of GBM cells. Notably, by searching online microRNA databases and in silico analysis, Notch1 and Notch2 were identified as potential target genes of miR-146a. Additionally, Notch1 has been validated as a target gene of miR-146a using a luciferase assay in GBM cells, and it was induced as a negative-feedback regulator to suppress tumor growth via the inhibition of Notch1 (17).

Based on the above evidence, the differential inhibitory effect of miR-146a on Notch1 and Notch2 was evaluated at various concentrations of the miRNA. Simultaneously, genotyping analyses were performed for the miR-146a rs2910164 polymorphism and their associations with the prognosis of GBM in 380 Chinese GBM patients were evaluated.

\section{Materials and methods}

Patients. A total of 380 patients with histologically confirmed GBM were recruited from the Department of Neurosurgery, The First Affiliated Hospital of Dalian Medical University (Dalian, China) between 2008 and 2012. Of this total, resected tumor specimens were available from 138 patients. All participants were ethnic Han Chinese individuals. The study was approved by investigational review committees at Dalian Medical University. Written informed consent was obtained from each participant. Demographic data and information regarding known and potential risk factors were obtained through interviewer administered questionnaires.

Biospecimen collection. Genomic DNA samples for genotyping were isolated from peripheral venous blood collected from all participants using a DNA extraction kit obtained from Tiangen Biotech Co. Ltd. (Beijing, China). A NanoPhotometer Ultraviolet/Vis Spectrophotometer (Implen GmbH, Munich, Germany) and $0.6 \%$ agarose electrophoresis were used to assess the concentration and purity of the DNA prior to its storage at $-20^{\circ} \mathrm{C}$ until future use.

Reverse transcription-quantitative polymerase chain reaction (RT-qPCR). Total RNA was obtained using the TRIzol one-step RNA isolation kit (Invitrogen Life Technologies, Carlsbad, CA, USA). The miR-146a, Notch1, Notch2 and U6 (internal control)-specific cDNA were synthesized from total RNA using gene-specific primers according to the TaqMan MicroRNA assay protocol (Applied Biosystems, Foster City, CA, USA). In a 96-well plate, $90 \mu 1$ master mix was added to
$2 \mu \mathrm{g}$ total RNA from each sample and run under the following thermal cycling conditions: $25^{\circ} \mathrm{C}$ for $10 \mathrm{~min}, 48^{\circ} \mathrm{C}$ for $30 \mathrm{~min}$ and $95^{\circ} \mathrm{C}$ for $5 \mathrm{~min}$. Relative quantification of target miRNA expression was conducted using the $\Delta \Delta$ cycle threshold $(\Delta \Delta \mathrm{Ct})$ method. Each sample was evaluated in triplicate and the raw data are presented as the relative quantity of target miRNA, normalized with respect to U6.

Genotyping of miR-146a rs2910164 SNP. Genotyping was performed as described previously (11). Briefly, DNA specimens were amplified using standard PCR protocols. The PCR products were purified with the ExoSAP-IT purification kit (USB Corporation, Cleveland, OH, USA) and were subsequently sent to the core sequencing lab located in Dalian Medical University for sequencing using the ABI sequencing system (PE Applied Biosystems, Foster City, CA, USA). The sequencing results were analyzed using Chromosome DNAstar software, version 12.1 (DNASTAR, Inc., Madison, WI, USA). The PCR primers used for miR-146a sequencing were: Forward, 5'-ATTTTACAGGGCTGGGACAG-3' and reverse, 5'-TCTTCCAAGCTCTTCAGCAG-3'.

Western blot analysis. Total proteins were isolated from U251 glioma cells (Type Culture Collection of the Chinese Academy of Sciences, Shanghai, China) that were transfected with scramble, miR-146a mimic or miR-146a inhibitor (Shanghai GenePharma Co., Ltd., Shanghai, China), respectively. The protein concentration was quantitatively measured prior to being loaded onto $10 \%$ SDS-polyacrylamide gel (Invitrogen Life Technologies) and subsequently transferred onto a polyvinylidene difluoride membrane (EMD Millipore, Billerica, MA, USA). The membrane was then sequentially incubated with rabbit polyclonal IgG anti-Notch1 (sc-9170) and anti-Notch2 (sc-5545) primary antibodies (1:2,000; $2 \mathrm{~h}$ at room temperature) and horseradish peroxidase-conjugated secondary antibody (sc-2371; 1:10,000; $1 \mathrm{~h}$ at room temperature) according to manufacturer's instructions. All antibodies were purchased from Santa Cruz Biotechnology, Inc. (Santa Cruz, CA, USA) and the signals were detected using an ECL chemifluorescence detection kit (Pierce Biotechnology, Inc., Rockford, IL, USA). The band density of specific proteins was densitometrically analyzed following normalization to the density of $\beta$-actin.

MTT assay. The growth rate of control and transfected U251 cells was determined using an MTT assay. Briefly, $4 \times 10^{3}$ cells/well were plated onto a 96-well plate. Levels of cell growth were measured each day for six consecutive days following plating, by adding $20 \mu \mathrm{l}(5 \mathrm{mg} \mathrm{MTT} / \mathrm{ml}$; Sigma-Aldrich, St. Louis, MO, USA) to the well and incubating for an additional $4 \mathrm{~h}$, prior to termination of the assay by lysing the cells with $200 \mathrm{ml}$ dimethyl sulfoxide (Sigma-Aldrich) for $5 \mathrm{~min}$. Optical density was measured in triplicate at $570 \mathrm{~nm}$ using a xMark Microplate Absorbance spectrophotometer (Bio-Rad Laboratories, Inc., Hercules, CA, USA) and expressed as a percentage of the control.

Transwell assay. Transwell filters (Corning Costar, Inc., Corning, NY, USA) were coated with Matrigel $(3.9 \mathrm{mg} / \mathrm{ml}$, 60-80 ml; BD Biosciences Discovery Labware, Bedford, MA, 
Table I. Clinicopathological and demographic characteristics of the patients involved in the study.

\begin{tabular}{|c|c|c|c|}
\hline Characteristic & $\begin{array}{l}\text { GG/GC } \\
(n=226)\end{array}$ & $\begin{array}{c}C C \\
(n=154)\end{array}$ & P-value \\
\hline Age (years \pm standard deviation) & $54.30 \pm 12.81$ & $58.11 \pm 9.63$ & 0.150 \\
\hline Gender (male: female) & $76: 150$ & 55:99 & 0.674 \\
\hline Smoking status (smoker: nonsmoker) & $101: 125$ & $63: 91$ & 0.465 \\
\hline
\end{tabular}
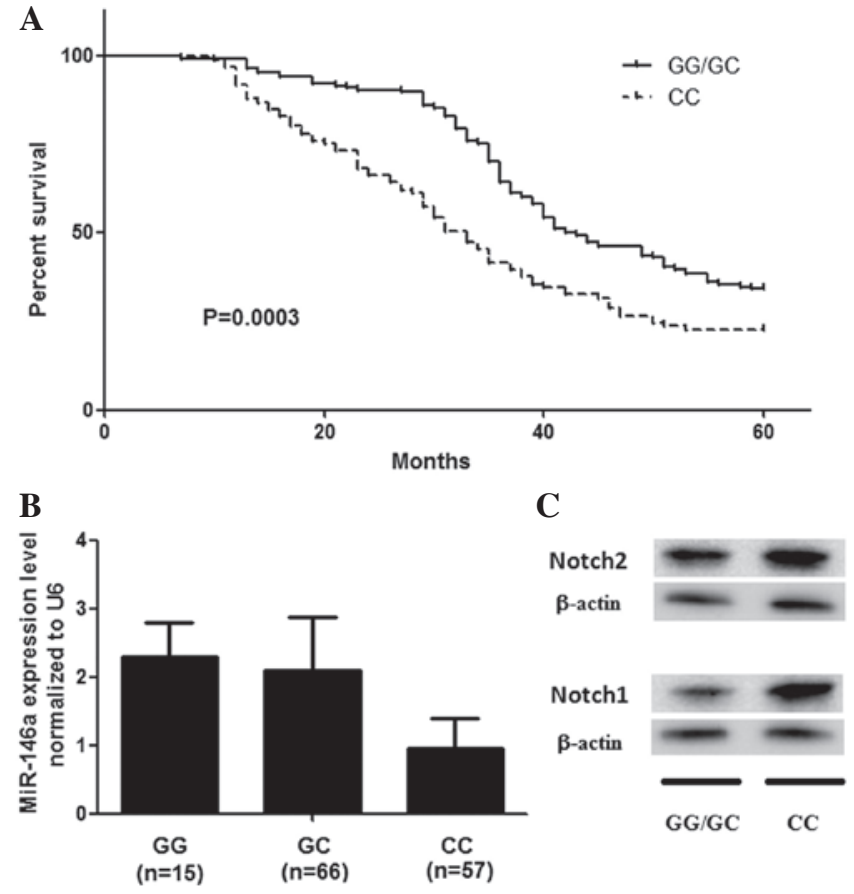

Figure 1. Evaluation of association between rs2910164 genotype, survival rate and miR-146a expression levels. (A) Kaplan-Meier survival curves for 380 GBM cases stratified by pre-miR $146 a$ rs2910164 genotype. The log-rank method was used to assess genotype-specific differences in survival distributions. (B) Expression levels of miR-146a of each $146 a$ rs2910164 genotype group in 138 GBM cases. (C) Protein expression levels of Notch1 and Notch2 in GG/GC and CC rs2910164 genotype groups in 138 GBM cases. GBM, glioblastoma multiforme. ${ }^{*} \mathrm{P}<0.05$.

USA) and incubated at $37^{\circ} \mathrm{C}$ for $30 \mathrm{~min}$, prior to solidification of the Matrigel. Matrigel is used as a surrogate for the major components of the extracellular matrix in tumor cell invasion. Transfected and control cells $\left(1 \times 10^{5}\right)$ suspended in $200 \mathrm{ml}$ serum-free Dulbecco's modified Eagle's medium (Invitrogen Life Technologies) were added to the upper chamber and the conditioned medium containing tumor cells was added into the lower chamber. Following $24 \mathrm{~h}$ of incubation at $37^{\circ} \mathrm{C}$ in $5 \% \mathrm{CO}_{2}$, the medium was removed from the upper chamber. The non-invaded cells on the upper surface of the inserted filter were gently scraped off with a wet cotton swab and the cells that had invaded the lower surface of the filter were fixed in methanol and counted. The quantity was expressed as a percentage of the control.

Statistical analysis. Fisher's exact $\chi^{2}$ test was used to compare the frequency distribution of age, gender and smoking status between high- and low-grade groups, if appropriate. The
Kaplan-Meier method was used to estimate overall survival (OS), defined as the length of time between study registration and a patient's mortality (of the patients who died). The OS periods were compared using the log-rank test. Univariate and multivariate Cox proportional hazard regression analyses were performed to estimate the effect of miR-146a polymorphisms on survival rate and the expression levels of miR-146 and Notch1 in the presence of known prognostic factors, including age, gender and smoking status. Data were analyzed using SPSS 19.0 software (IBM, Armonk, NY, USA) and $\mathrm{P}<0.05$ was considered to indicate a statistically significant difference.

\section{Results}

Demographic and clinicopathological characteristics of the participants. In the present study a total of 380 participants (198 males and 82 females), with a mean age of 56.92 years, were recruited. Information regarding the known risk factors of GBM prognosis, including age, gender and smoking status are described in Table I.

Patients with the rs2910164 CC polymorphism have a reduced survival rate. The genotype frequency of the SNP amongst the study population fit the Hardy-Weinberg equilibrium. Initially, the association between the SNP and the survival rate in the GBM patients was evaluated. As shown in Fig. 1A, participants carrying the rs2910164 CC genotype had a significantly decreased survival rate compared with that of the GG and GC genotypes, following adjustments for age, gender and smoking status $(\mathrm{P}=0.002)$. The effect of the rs2910164 $\mathrm{C}$ allele on survival rate indicated a recessive pattern in GBM patients (data not shown).

Patients carrying the rs2910164 CC genotype exhibit increased miR-146a expression. The mRNA expression level of miR-146a was also measured in the 138 patients whose tumor specimens were available and its association with the genetic variant was evaluated. As shown in Fig. 1B, the expression levels of miR-146a in those who carried the rs2910164 $\mathrm{CC}$ genotype were significantly higher than those of the $\mathrm{GC}$ and GG carriers $(\mathrm{P}<0.001)$. The number of rs2910164 $\mathrm{G}$ alleles revealed a dominant effect on the expression of miR-146a, as shown in Fig. 1. Furthermore, Notch1 and Notch2 were found to be differentially expressed in individuals with the GG/GC and CC genotypes (Fig. 1C).

Notch1 and 2 are targets of miR-146a and miR-146a expression levels affect the Notchl/2 ratio. The candidate target genes of miR-146a were further computationally screened 
A

Notch2 WT 3' UTR

$$
\begin{gathered}
\text { 5' TTTTGGTTTGAATGACAGTTCTCA 3' } \\
|||||||| \mid \\
\text { 3' TTGGGTACCTTAAGTCAAGAGU 5, }
\end{gathered}
$$

$\operatorname{miR}-146 \mathrm{a}$

Notch2 Ilut 3' UTR 5' TTTTGGTTGAATGAGTCAAGAGU 3
miR-146a versus Notch2 3' UTR

\begin{tabular}{|c|c|c|}
\hline Notch1 IPT 3' UTR & 5 & $\begin{array}{c}\text { CAAGAAAATAAACTAGTTCTCA } 3^{\prime} \\
\|\|\|\| \|\end{array}$ \\
\hline $\operatorname{miR}-146 a$ & 3 & TTGGGTACCTTAAGTCAAGAGU 5 \\
\hline Notch1 IIut 3' UTR & 5 & CAAGAAAATAAACTTCAAGAGU 3 \\
\hline
\end{tabular}

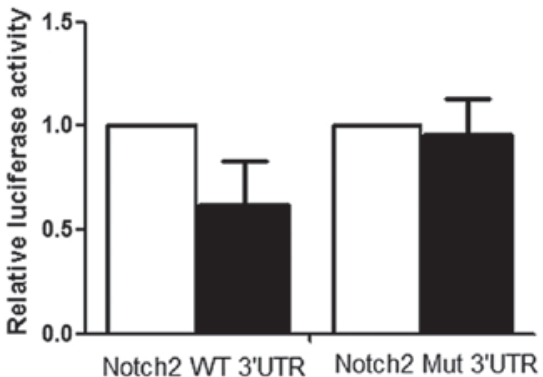

B

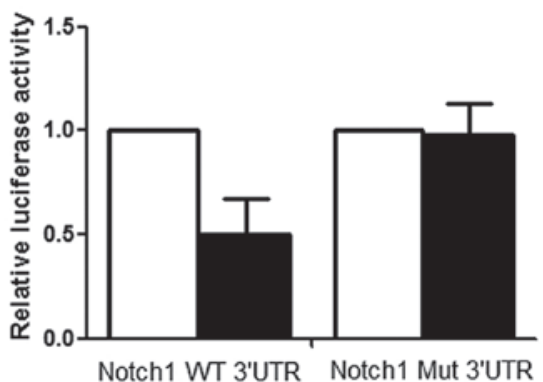

Figure 2. Identification of miR-146a target genes. (A) Notch1 is a direct target of miR-146a in glioma cells. Left, schematic representation of the miR-146a-binding sequence in the 3'-UTR of Notch1 mRNA. Mutations were generated in the miR-146a-binding sequence of the Notch1 3'-UTR as indicated; Right, analysis of luciferase activity in U251 cells $48 \mathrm{~h}$ after co-transfection with the control Renilla luciferase expression construct pRL-TK and a Firefly luciferase reporter plasmid containing wild-type or mutant Notch1 3'-UTR (indicated as WT or MUT on the axis, respectively). Firefly luciferase activity in each sample was normalized to Renilla activity, and expressed relative to the normalized luciferase activity of control cells $(\mathrm{P}<0.05)$. (B) Notch2 is a direct target of miR-146a in glioma cells. Left, schematic representation of the miR-146a-binding sequence in the 3'-UTR of Notch2 mRNA. Mutations were generated in the miR-146a-binding sequence of the Notch2 3'-UTR as indicated; Right, analysis of luciferase activity in U251 cells $48 \mathrm{~h}$ after co-transfection with the control Renilla luciferase expression construct pRL-TK and a Firefly luciferase reporter plasmid containing either the wild-type or mutant Notch2 3'-UTR (indicated as WT or MUT on the axis, respectively). Firefly luciferase activity in each sample was normalized to Renilla activity, and expressed relative to the normalized luciferase activity of control cells $(\mathrm{P}<0.05)$. miR, microRNA; UTR, untranslated region; WT, wildtype; MUT, mutant. " $\mathrm{P}<0.05$.

A

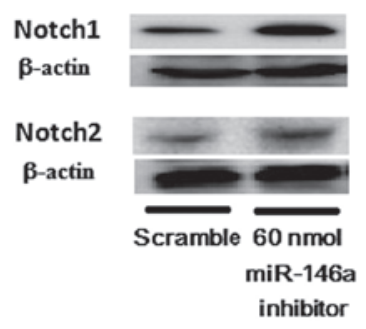

Notch1

$\beta$-actin

Notch2

B-actin

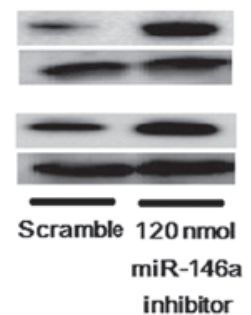

B

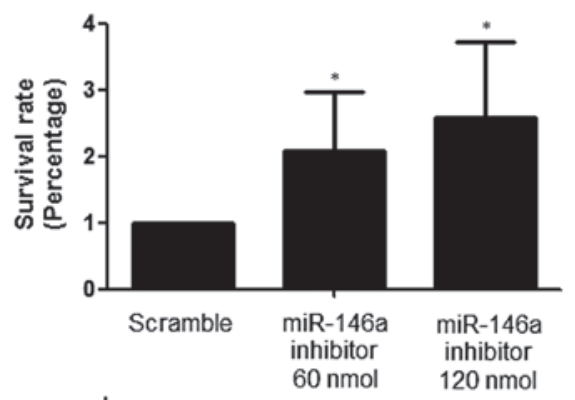

C

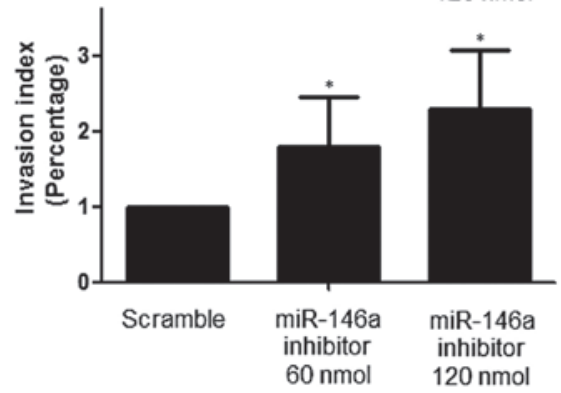

Figure 3. In vitro study of proliferation and invasive ability of U251 cells transfected with miR-146a mimic. (A) Suppression of Notch1 and Notch2 by 60

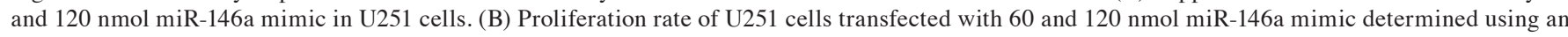
MTT assay. (C) Invasive ability of U251 cells transfected with 60 and $120 \mathrm{nmol}$ miR-146a mimic was examined using a Transwell assay. miR, microRNA.

and identified using TargetScan Release 5.1 (http://www. targetscan.org). Notch1 and Notch2 were identified as potential target genes of miR-146a, which was subsequently confirmed using a luciferase assay, as shown in Fig. 2. Considering the opposing roles of Notch1 and Notch2 in gliomagenesis, the direct effect of miR-146a on cell behavior, as well as the expression levels of Notch1 and Notch2, were investigated using western blot analysis and the results revealed that transfection with miR-146a mimic induced a significant downregulation of Notch1 and Notch2 expression to differing extents, presenting 
A

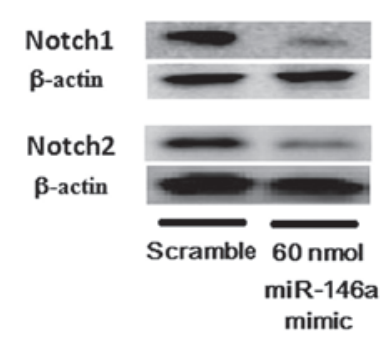

Notch1

B-actin

Notch2

$\beta$-actin

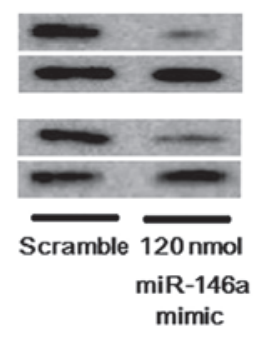

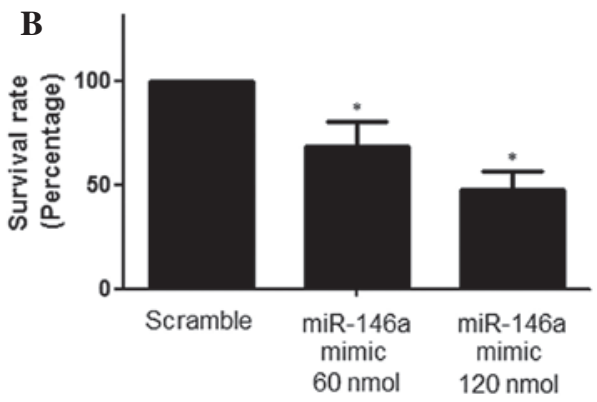

C

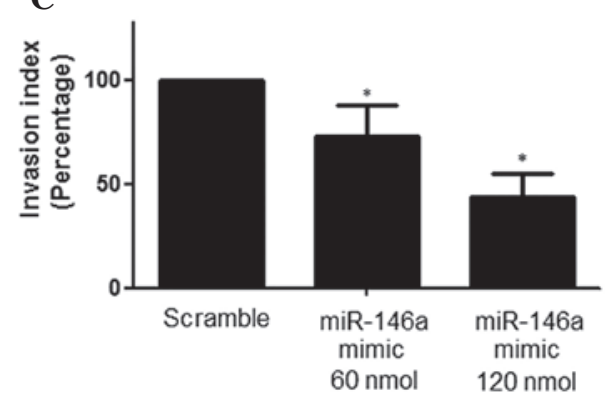

Figure 4. In vitro study of proliferation and invasive ability in U251 cells transfected with miR-146a inhibitor. (A) Suppression of Notch1 and Notch2 by 60 and $120 \mathrm{nmol}$ miR-146a inhibitor in U251 cells. (B) Proliferation rate of U251 cells transfected with 60 and $120 \mathrm{nmol}$ miR-146a inhibitor determined using an MTT assay. (C) Invasive ability of U251 cells transfected with 60 and $120 \mathrm{nmol}$ miR-146a inhibitor examined using a transwell assay. miR, microRNA.

a stepwise reduction in the Notch1/Notch2 ratio coupled with an ascending concentration of the miR-146a mimic (Fig. 3A) and suppressed the growth and invasion of U251 cells (Fig. 3B). Consistent with these results, the introduction of an miR-146a inhibitor resulted in a marked upregulation of Notch1 and Notch2 expression, with a corresponding stepwise change in the Notch1/Notch2 ratio (Fig. 4A) and promoted cell proliferation and invasion (Fig. 4B and C) with various concentrations of the inhibitor.

Tissue samples in the CC group exhibit greater Notchl and 2 expression compared with the GG/GC group. Tissue samples from 138 glioma patients were also collected and the expression patterns of miR-146a, Notch1 and Notch2 were determined using RT-qPCR and western blot analysis in two genotypic groups. The expression levels of miR-146a were higher, and Notch1 and Notch2 were lower in the GG/GC group than those in the $\mathrm{CC}$ group, and the Notch1/Notch2 ratio was significantly higher in the $\mathrm{CC}$ group than that in the GG/GC group (data not shown).

\section{Discussion}

In the present study, the association between a common miR-146a polymorphism (rs2910164) and glioma prognosis was evaluated in a Chinese population of 380 GBM cases. It was identified that the CC genotype was associated with a decreased survival rate amongst patients with GBM.

Permuth-Wey et al (19) reported that the rs2910164 $\mathrm{CC} / \mathrm{GC}$ genotypes were associated with an increased risk of glioma, particularly among older individuals, and that the $\mathrm{C}$ allele was associated with a decreased survival rate among patients with glioblastoma. Xu et al (20) demonstrated that the rs2910164 CC genotype was associated with a decreased risk of prostate cancer. Xu et al (22) observed that the GG genotype was associated with an increased risk of hepatocellular carcinoma. Jazdzewski et al (11) demonstrated that the GC genotype was associated with an increased risk of papillary thyroid cancer compared with that of the GC/CC genotypes. Guo et al (23) identified an association between the GG genotype and an increased risk of esophageal squamous cell carcinoma, particularly among smokers. The $\mathrm{C}$ allele carriers were reported to be more susceptible to gastric cancer in a Japanese population (24), and conversely the $\mathrm{C}$ allele was found to be protective against gastric cancer in a Chinese population (25). Inconsistent results regarding the association between the rs2910164 miR-146a genotype and cancer risk may indicate a heterogeneous tumor etiology or differences in the ethnicity of investigated populations.

In the present study, the rs2910164 CC genotype was associated with the risk for high-grade glioma. However, unlike previously reported age-specific effects for this SNP (25-27), the association was comparable when stratified with age. Survival analyses based on the rs2910164 CC genotype indicated a decreased survival rate in the patients with high-grade glioma, with a stepwise decrease in miR-146a expression levels. This trend was also identified in low-grade gliomas, but the rs2910164 genotypes did not influence the survival rate of this population.

Jazdzewski et al (11) demonstrated that the $\mathrm{C}$ allele of rs2910164 in the pre-miR-146a sequence interfered with the correct processing and expression of the miRNA and induced a 1.8-fold reduction in mature miR-146a compared with that of the $\mathrm{G}$ allele (27). The SNP was located at position +60 relative to the first nucleotide on the passenger strand of pre-miR-146a, with the $\mathrm{C}$ allele being hypothesized to lead to mispairing within the mature hairpin (11). It has been suggested that the rs2910164 genotype may contribute to carcinogenesis via mediation of miR-146a interactions with key target genes (14). Jazdzewski et al (11) also demonstrated that the $\mathrm{C}$ allele 
compromised the ability of miR-146a to bind with HeLa cell nuclear proteins and resulted in inefficient inhibition of the miR-146a target genes TRAF6 and IRAK1. Peng et al (28) demonstrated that TRAF6 was involved in the potentiation of growth, proliferation, invasion and migration of glioma cells (U-87MG cell line), and it exerted an inhibitory effect on the apoptosis of glioma cells by activating NF- $\mathrm{BB}$. Concurrently, Funakoshi et al (29) observed that overexpression of TRAF6 enhanced interleukin-1 mediated $N F-\kappa B$ and activator protein 1 activation. TRAF6 is also able to upregulate hypoxia inducible factor-1 $\alpha$ expression and promote tumor angiogenesis and growth (30). Additionally, TRAF6 has been reported to have a significant oncogenic role in other types of cancer, including esophageal squamous cell carcinoma, non-small cell lung cancer and multiple myeloma, by either activating $\mathrm{NF}-\kappa \mathrm{B}$ and Janus kinase/signal transducer and activator of transcription pathways or via downregulation of activated caspase 3 and cleaved poly ADP ribose polymerase, and upregulation of c-Jun, B cell lymphoma 2 and c-Myc (31-33).

Notch signaling has been demonstrated to cumulate oncogenic activities involved in glioma proliferation, apoptosis inhibition and invasion, in addition to its functions in the maintenance of non-neoplastic neural stem cells and neovascularization (34). Notch1 is a validated target of miR-146a, and miR-146a has been observed to be induced as a negative-feedback regulator to suppress glioma growth via inhibition of Notch1 (17). Chen et al (15) demonstrated increased Notch1 expression levels in glioma and suggested that this increase may be associated with tumor progression. Zhang et al (16) demonstrated that Notch activation may stimulate the AKT pathway and subsequently activate $\beta$-catenin and $\mathrm{NF}-\kappa \mathrm{B}$ signaling in glioma cells. By contrast, another major member of Notch family, Notch2, was found to have a different role in the control of glioma cell activities. Upregulation of Notch2 resulted in the induction of apoptosis, as well as the suppresion of cell growth and invasion, similarly to the results of silencing Notch1 (21). In addition, high levels of Notch2 indicated a favorable prognosis in all subtypes of glioma (35). In the present study, it was verified that Notch1 and Notch2 were target genes of miR-146a in glioma cells and that the introduction of miR-146a suppressed Notch1 and Notch2 expression. It was therefore hypothesized that Notch1 and Notch2 maintain a balance in regulating cell behavior and the altered level of miR-146 induced by the rs2910164 polymorphism alters the balance between the two Notch family members, resulting in changes in cell behavior and malignancy transformation. The hypothesis was investigated by transfecting miR-146a mimic into U251 cells, and confirmed by the observation that overexpression of miR-146a induced a significant downregulation of Notch1 and Notch 2 to differing extents, presenting a stepwise decrease in Notch1/Notch 2 ratio with ascending concentration of the miR-146a mimic. Conversely, the introduction of miR-146a inhibitor resulted in marked upregulation of Notch 1 and Notch2 expression with a corresponding stepwise increase of Notch1/Notch2 ratio at various concentrations of the inhibitor. Additionally, the expression pattern of miR-146a, Notch1 and Notch2 in the GG/GC group versus the CC group was consistently in concordance with the aforementioned in vitro functional studies.
In the present study, the rs2910164 C allele was found to be associated with a decreased survival rate, indicating that this variant may contribute to tumor progression by inducing loss-of-function of miR-146a. Confirmation of the current association in a larger population is required. Knowledge of the inherited variation in miRNA-associated genes may aid the identification of high-risk populations and the development of diagnostic, prognostic and therapeutic strategies in order to reduce the burden of gliomas and other malignancies.

\section{References}

1. Louis DN, Ohgaki H, Wiestler OD, Cavenee WK, Burger PC, Jouvet A, Scheithauer BW and Kleihues P: The 2007 WHO classification of tumours of the central nervous system. Acta Neuropathol 114: 97-109, 2007.

2. Hemminki $\mathrm{K}$ and $\mathrm{Li} \mathrm{X}$ : Familial risks in nervous system tumors. Cancer Epidemiol Biomarkers Prev 12: 1137-1142, 2003.

3. Scheurer ME, Etzel CJ, Liu M, El-Zein R, Airewele GE, Malmer B, Aldape KD, Weinberg JS, Yung WK and Bondy ML: Aggregation of cancer in first-degree relatives of patients with glioma. Cancer Epidemiol Biomarkers Prev 16: 2491-2495, 2007.

4. Bondy ML, Scheurer ME, Malmer B, Barnholtz-Sloan JS, Davis FG, Il'yasova D, Kruchko C, McCarthy BJ, Rajaraman P, Schwartzbaum JA, et al: Brain tumor epidemiology: consensus from the brain tumor epidemiology consortium. Cancer 113: 1953-1968, 2008.

5. Shete S, Hosking FJ, Robertson LB, Dobbins SE, Sanson M, Malmer B, Simon M, Marie Y, Boisselier B, Delattre JY, et al: Genome-wide association study identifies five susceptibility loci for glioma. Nat Genet 41: 899-904, 2009.

6. Wrensch M, Jenkins RB, Chang JS, Yeh RF, Xiao Y, Decker PA, Ballman KV, Berger M, Buckner JC, Chang S, et al: Variants in the CDKN2B and RTEL1 regions are associated with high-grade glioma susceptibility. Nat Genet 41: 905-908, 2009.

7. Egan KM, Thompson RC, Nabors LB, Olson JJ, Brat DJ,Larocca RV, Brem S, Moots PL, Madden MH, Browning JE and Ann Chen Y: Cancer susceptibility variants and the risk of adult glioma in a US case-control study. J Neurooncol 104: 535-542, 2011.

8. Calin GA and Croce CM: MicroRNA signatures in human cancers. Nat Rev Cancer 6: 857-866, 2006.

9. Ryan BM, Robles AI and Harris CC: Genetic variation in microRNA networks: the implications for cancer research. Nat Rev Cancer 10: 389-402, 2010.

10. Yang Q, Jie Z, Ye S, Li Z, Han Z, et al: Genetic variations in miR-27a gene decrease mature miR-27a level and reduce gastric cancer susceptibility. Oncogene 33: 193-202, 2014.

11. Jazdzewski K, Murray EL, Franssila K, Jarzab B, Schoenberg DR, et al: Common SNP in pre-miR-146a decreases mature miR expression and predisposes to papillary thyroid carcinoma. Proc Natl Acad Sci USA 105: 7269-7274, 2008.

12. Turner JD, Williamson R, Almefty KK, Nakaji P, Porter R, Tse V and Kalani MY: The many roles of microRNAs in brain tumor biology. Neurosurg Focus 28: E3, 2010.

13. Duan R, Pak C and Jin P: Single nucleotide polymorphism associated with mature miR-125a alters the processing of pri-miRNA. Hum Mol Genet 16: 1124-1131, 2007.

14. Chae YS, Kim JG, Lee SJ, Kang BW, Lee YJ, Park JY, Jeon HS, Park JS and Choi GS: A miR-146a polymorphism (rs2910164) predicts risk of and survival from colorectal cancer. Anticancer Res 33: 3233-3239, 2013.

15. Chen L, Zhang R, Li P, Liu Y, Qin K, Fa ZQ, Liu YJ, Ke YQ and Jiang XD: P53-induced microRNA-107 inhibits proliferation of glioma cells and down-regulates the expression of CDK6 and Notch-2. Neurosci Lett 534: 327-332, 2013.

16. Zhang X, Chen T, Zhang J, Mao Q, Li S, Xiong W, Qiu Y, Xie Q and Ge J: Notch1 promotes glioma cell migration and invasion by stimulating $\beta$-catenin and NF- $\kappa$ B signaling via AKT activation. Cancer Sci 103: 181-190, 2012.

17. Mei J, Bachoo R and Zhang CL: MicroRNA-146a inhibits glioma development by targeting Notch1. Mol Cell Biol 31: 3584-3592, 2011.

18. Treanor LM, Volanakis EJ, Zhou S, Lu T, Sherr CJ and Sorrentino BP: Functional interactions between Lmo2, the Arf tumor suppressor and Notch1 in murine T-cell malignancies. Blood 117: 5453-5462, 2011. 
19. Permuth-Wey J, Thompson RC, Burton Nabors L, Olson JJ, Browning JE, Madden MH, Ann Chen Y and Egan KM: A functional polymorphism in the pre-miR-146a gene is associated with risk and prognosis in adult glioma. J Neurooncol 105: 639-646, 2011.

20. Xu B, Feng NH, Li PC, Tao J, Wu D, Zhang ZD, Tong N Wang JF, Song NH, Zhang W, Hua LX and Wu HF: A functional polymorphism in Pre-miR-146a gene is associated with prostate cancer risk and mature miR-146a expression in vivo. Prostate 70 467-472, 2010.

21. Xu P, Zhang A, Jiang R, Qiu M, Kang C, Jia Z, Wang G, Han L, Fan $\mathrm{X}$ and $\mathrm{Pu} \mathrm{P}$ : The different role of Notch1 and Notch2 in astrocytic gliomas. PLoS One 8: e53654, 2013.

22. Xu T, Zhu Y, Wei QK, Yuan Y, Zhou F, Ge YY, Yang JR, Su H and Zhuang SM: A functional polymorphism in the miR-146a gene is associated with the risk for hepatocellular carcinoma. Carcinogenesis 29: 2126-2131, 2008.

23. Guo H, Wang K, Xiong G, Hu H, Wang D, Xu X, Guan X, Yang K and Bai Y: A functional varient in microRNA-146a is associated with risk of esophageal squamous cell carcinoma in Chinese Han. Fam Cancer 9: 559-603, 2010.

24. Okubo M, Tahara T, Shibata T, Yamashita H, Nakamura M, Yoshioka D, Yonemura J, Kamiya Y, Ishizuka T, Nakagawa Y, et al: Association study of common genetic variants in pre-microRNAs in patients with ulcerative colitis. J Clin Immunol 31: 69-73, 2011.

25. Zeng Y, Sun QM, Liu NN, Dong GH, Chen J, Yang L and Wang B: Correlation between premiR-146a C/G polymorphism and gastric cancer risk in Chinese population. World J Gastroenterol 16: 3578-3583, 2010.

26. Shen J, Ambrosone CB, DiCioccio RA, Odunsi K, Lele SB and Zhao H: A functional polymorphism in the miR-146a gene and age of familial breast/ovarian cancer diagnosis. Carcinogenesis 29: 1963-1966, 2008.
27. Iyer A, Zurolo E, Prabowo A, Fluiter K, Spliet WGM, van Rijen PC, Gorter JA and Aronica E: MicroRNA-146a: a key regulator of astrocyte-mediated inflammatory response. PLoS One 7: e44789, 2012.

28. Peng Z, Shuangzhu Y, Yongjie J, Xinjun Z and Ying L: TNF receptor-associated factor 6 regulates proliferation, apoptosis, and invasion of glioma cells. Mol Cell Biochem 377: 87-96, 2013.

29. Funakoshi M, Sonoda Y, Tago K, Tominaga S and Kasahara T: Differential involvement of p38 mitogen-activated protein kinase and phosphatidyl inositol 3-kinase in the IL-1-mediated NF-kappaB and AP-1 activation. Int Immunopharmacol 1: 595-604, 2001

30. Sun H, Li XB, Meng Y, Fan L, Li M and Fang J: TRAF6 upregulates expression of HIF-1 $\alpha$ and promotes tumor angiogenesis. Cancer Res 73: 4950-4959, 2013.

31. Yao F, Han Q, Zhong C and Zhao H: TRAF6 promoted the tumorigenicity of esophageal squamous cell carcinoma. Tumour Biol 34: 3201-3207, 2013.

32. Liu H, Tamashiro S, Baritaki S, Penichet M, Yu Y, Chen H, Berenson $\mathrm{J}$ and Bonavida B: TRAF6 activation in multiple myeloma: a potential therapeutic target. Clin Lymphoma Myeloma Leuk 12: 155-163, 2012.

33. Liu H, Zhang T, Ye J, Li H, Huang J, Li X, Wu B, Huang X and Hou J: TNF receptor-associated factor 6 in advanced non-small cell lung cancer: clinical and prognostic implications. J Cancer Res Clin Oncol 138: 1853-1863, 2012.

34. Hori K, Sen A and Artavanis-Tsakonas S: Notch signaling at a glance. J Cell Sci 126: 2135-2140, 2013

35. Boulay JL, Miserez AR, Zweifel C, Sivasankaran B, Kana V, Ghaffari A, Luyken C, Sabel M, Zerrouqi A, Wasner M, et al: Loss of NOTCH2 positively predicts survival in subgroups of human glial brain tumors. PLoS One 2: e576, 2007. 